\title{
Bone Response
}

National Cancer Institute

\section{Source}

National Cancer Institute. Bone Response. NCI Thesaurus. Code C132455.

An assessment of the bone response of a disease to a therapy. 